\title{
Influences relatives des agents hydrodynamiques sur la mobilité des sédiments non-cohésifs des Pertuis Charentais
}

\author{
Déborah Idier ${ }^{1}$, Emmanuel Romieu ${ }^{1}$, Rodrigo Pedreros ${ }^{1}$ \\ ${ }^{1}$ BRGM, Service Aménagement et Risques Naturels, 3 av. C. Guillemin, BP \\ 6009, 45060 Orléans cédex 2 \\ d.idier@brgm.fr, e.romieu@brgm.fr,r.pedreros@brgm.fr
}

\begin{abstract}
Résumé :
L'influence relative des vagues, courants de marée et courants induits par le vent sur la mobilité sédimentaire est étudiée en domaine de plateforme interne macrotidale (au large des Pertuis Charentais). Des mesures hydrodynamiques avaient déjà montré le rôle important de la houle dans la mise en mouvement des sables entre l'île de Ré et l'île d'Oléron. Même si la méthode présentée comporte des limites, la modélisation mise en place, basée sur le code de vagues SWAN et le code hydrodynamique 2DH MARS, permet d'étendre spatialement la connaissance des rôles relatifs des vagues (hors courants de houle) et des courants sur la mise en mouvement des sédiments non cohésifs à l'échelle des Pertuis Charentais.
\end{abstract}

\begin{abstract}
:
This paper investigates the relative influence of waves, wind-induced current and tidal current on the sediment mobility of a macro-tidal environment belonging to the inner shelf seaward of the Pertuis Charentais (France). Past studies, based on hydrodynamic measurements already showed the dominant role of swells in the initiation of the motion of sand in an area located between the Ré and Oléron islands. Even if the method has some limits, using wave (SWAN) and 2DH hydrodynamic (MARS) modelling, we extend spatially the knowledge of the relative contribution of waves (without consideration of wave-induced currents), tides and wind-induced current in the non-cohesive sediment mobility.
\end{abstract}

\section{Mots-clés :}

Pertuis Charentais - courants - vagues - plateau continental - mobilité sédiment - modélisation - non-cohésif. 


\section{$1 \quad$ Introduction}

Les fonds marins, en particulier dans les zones de plateforme continentale interne, sous régime macro-tidal, sont soumis à l'action des courants de marée et de la houle. L'analyse des processus de transport sédimentaire passe par l'estimation de l'influence de chacun de ces deux processus. L'impact relatif théorique de la houle et des courants de marée sur les sédiments de la plate-forme du Golfe de Gascogne a déjà fait l'objet d'une étude (BARTHE et CASTAING, 1989), basée sur des mesures hydrodynamiques d'une part, et des abaques de mise en mouvement et de transport de sédiments d'autre part. Par ailleurs, l'influence des composantes de courants de marée, de houle, de mer de vent et de courants résiduels sur la mise en mouvement et le transport des sédiments a été étudiée par IDIER et al. (2006), à partir de mesures hydrodynamiques réalisées au niveau du seuil inter-insulaire, au large du Pertuis d'Antioche. Enfin, des travaux de modélisation hydrodynamiques ont été réalisés. Les courants de marée ont été modélisés dans la passe de Maumusson (BERTIN et al., 2005), ainsi qu'à l'échelle des Pertuis et des îles (STANISIERE et al., 2006). Des travaux ont aussi été menés sur la modélisation des vagues dans cette zone (BERTIN et al., 2008). Cependant, peu d'études ont été menées sur les rôles relatifs de la mise en mouvement des sédiments par les vagues et courants.

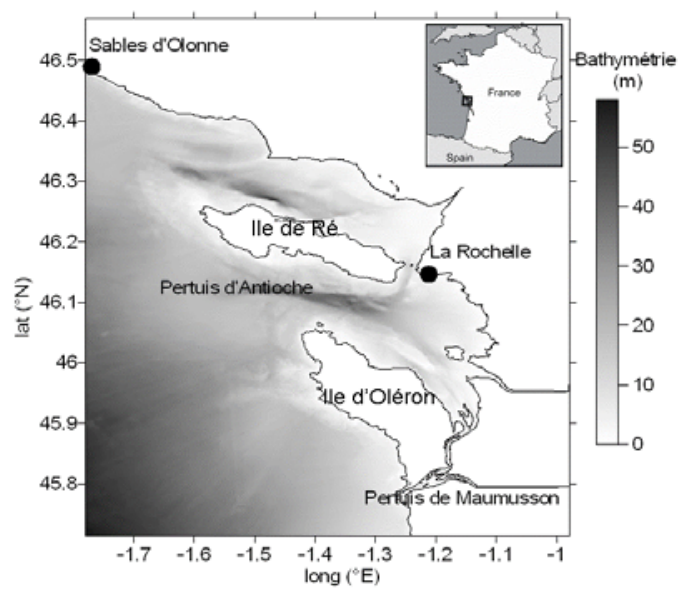

Figure 1 - Pertuis Charentais : bathymétrie. MNT issu de données bathymétriques SHOM, GEBCO, et IFREMER.

Le but de ce travail est de spatialement étendre la connaissance de l'influence des différentes composantes hydrodynamiques dans la mobilité des sédiments non-cohésifs, à l'échelle des Pertuis, en s'appuyant sur l'utilisation de modèles de vagues et de courants. Ces travaux s'effectuant à l'échelle des Pertuis, la résolution minimale des différents modèles mis en place et, donc des phénomènes étudiées, est de 200 à $300 \mathrm{~m}$ et nous nous intéressons à la mobilité sédimentaire induite avant les zones de déferlement (e.g. pour des hauteurs d'eau minimale de l'ordre de 5 à $10 \mathrm{~m}$ ). Les phénomènes comme les courants de houle, de retour ou 
de dérive ne sont donc pas considérés dans la présente étude.

Tout d'abord, la zone et la période d'étude sont présentées. Ensuite, les deux modèles sont décrits. Enfin, la mobilité sédimentaire est estimée.

\section{Zone et période d'étude}

Le site d'étude s'étend du pertuis de Maumusson au Sud jusqu'aux Sables d'Olonne au Nord, et des Sables d'Olonne à l'Ouest jusqu'au continent à l'Est. D'après l'atlas de courants du SHOM, les courants de marée intégrés sur la verticale sont de l'ordre de $0,45 \mathrm{~m} / \mathrm{s}$ et le marnage est de $5,1 \mathrm{~m}$ en vive-eau moyenne au niveau du seuil interinsulaire. En termes sédimentaires (SHOM, 2000), et de manière très schématique, de la côte vers le large, on rencontre tout d'abord des vases situées entre la côte et les deux îles, jusqu'à la baie de 1'Aiguillon au Nord. Ensuite, le long de la façade Ouest des îles, des platiers rocheux sont présents. Enfin, plus au large, la couverture sédimentaire est composée principalement de sables et de sables graveleux (pour la partie la plus externe), sauf au large du Sud-Ouest de l'île d'Oléron, où il existe une vasière.

IDIER et al. (2006) ont montré que, par $23 \mathrm{~m}$ de fond, au niveau du seuil interinsulaire, les houles (plutôt que la mer de vent) permettaient la mise en mouvement des sables, qui peuvent ensuite être transportés par les courants. Ils montrent aussi que des sables de 0,2 mm seraient ainsi transportés pendant $92 \%$ de la durée de la mesure, tandis que les graviers ne seraient que sporadiquement mis en mouvement, en particulier lors de conjonctions vive-eau - tempête.

Ces résultats ont été obtenus pour la période du 5 au 27 novembre 2002. C'est cette période de modélisation qui est choisie pour la présente étude. Pendant cette période, les coefficients de marée étaient compris entre 36 et 112. Trois épisodes de tempêtes ont eu lieu : le 07, les 13-14 et les 20-21 novembre, avec des vitesses de vent atteignant $15-20 \mathrm{~m} / \mathrm{s}$. Pendant la dernière période, la hauteur significative des vagues mesurée au niveau du seuil interinsulaire atteint plus de $6 \mathrm{~m}$, pour une période pic de $18 \mathrm{~s}$ (IDIER et al., 2006).

\section{$3 \quad$ Modélisation des vagues}

\subsection{Description et mise en place}

La modélisation des vagues s'appuie sur le code spectral SWAN qui est un modèle numérique de troisième génération, à différences finies, développé par l'université de Delft (Pays-Bas). L'évolution du spectre de vague y est décrite par l'équation spectrale de conservation de l'action d'onde (BOOIJ et al., 2004). Pour modéliser la zone des Pertuis, deux emboitements (Figure 2) sont mis en place ayant des tailles de maille allant de $1 \mathrm{~km}$ (B0) à $300 \mathrm{~m}$ (B1) (PEDREROS et $a l ., 2006)$. Les données bathymétriques utilisées pour construire les Modèles 


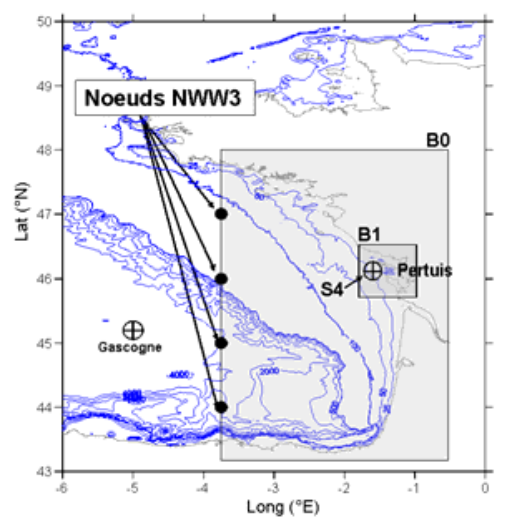

Figure 2 - Emprise du modèle de vagues et emboitements BO et B1.

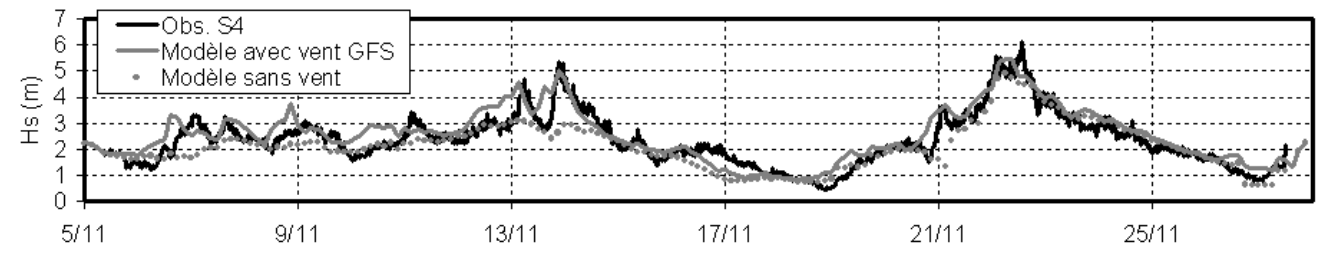

Figure 3 -Mesure et résultats du modèle SWAN au point S4.

Numériques de Terrain de chaque boite sont les données: GEBCO, SHOM et IFREMER. En termes de données de conditions aux limites, quatre nœuds du modèle NWW3 (GFS-NOAA) sont utilisés et fournissent les triplets (hauteur significative, période pic et direction pic), sur la frontière Ouest de la boite B0 (Figure 2). Les conditions météorologiques sont prises en compte sur toute l'emprise de la boite B0, en utilisant les données GFS. En termes de niveaux d'eau, le niveau de référence choisi dans les calculs SWAN est le niveau de la marée à la Rochelle (prédictions SHOM).

\subsection{Validation}

Ce modèle a été validé par rapport aux mesures réalisées au point S4 en octobrenovembre 2002 (PEDREROS et al., 2006). La Figure 3 montre tout d'abord l'influence de la prise en compte du vent dans la simulation. En effet, en négligeant le vent, le modèle sous-estime la hauteur des vagues d'un facteur pouvant atteindre 2 (exemple : le 14/11/2002). Le vent soufflant sur les emprises B0 et B1 doit donc être pris en compte. On observe un bon accord entre observations et modèle avec prise en compte du vent (Figure 3).

\section{$4 \quad$ Modèle de courants (ondes longues)}

\subsection{Description et mise en place}

La modélisation des ondes longues (marée et ondes de tempêtes) s'appuie sur le code MARS-2DH (équations de Saint-Venant). Ce code est développé par l'IFREMER et basé sur la méthode des différences finies. La technique de 
modélisation emboitée est utilisée, avec une boite de grande emprise R0 (taille de maille : $5500 \mathrm{~m}$ ) couvrant le Golfe de Gascogne et la Manche, jusqu'à une boite R3 (taille de maille : $200 \mathrm{~m}$ ) d'emprise couvrant l'île de Ré et l'île d'Oléron (Figure 4). Les données bathymétriques utilisées pour construire les Modèles Numériques de Terrain de chaque boite sont les données : GEBCO, SHOM et des levés spécifiques (LIDAR IFREMER par exemple dans les Pertuis).

Les conditions aux limites sont issues des constantes de marée FES2004 (LYARD et al., 2006). En cohérence avec la modélisation des vagues à l'aide du code SWAN, les données météorologiques utilisées pour la modélisation des ondes de tempête sont les données GFS au point $\left(46^{\circ} \mathrm{N} ; 2.5^{\circ} \mathrm{W}\right)$. Ces conditions de vent sont considérées comme uniformes sur toute la zone de modélisation.

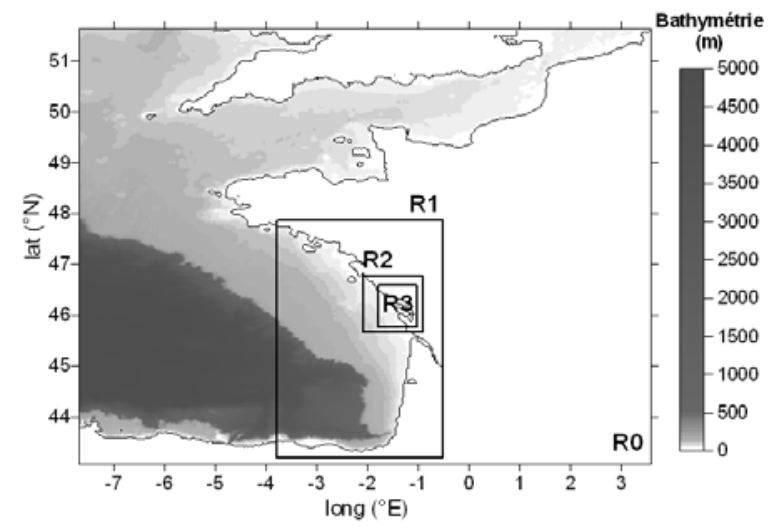

Figure 4-Emprise du modèle de courants. R0, $R 1, R 2$ et $R 3$ sont les différents emboitements du modèle.

\subsection{Validation}

En termes de reproduction de la marée (niveau d'eau et courants), le modèle a été comparé :

- aux prédictions de hauteur d'eau du SHOM aux ports de La Rochelle, à l'ile d'Aix et à la pointe de Gatseau sur l'ile d'Oléron pour une marée de viveeau moyenne de coefficient 95 (24 janvier 2004)

- aux observations de la campagne de mesure effectuée en octobre-novembre 2002, traitées avec le logiciel T-Tide pour extraire la composante de marée du signal (IDIER et al., 2006)

La validation de la marée pour deux cycles de marée de vive-eau moyenne montre des écarts rms de cote de la surface libre allant de $9 \mathrm{~cm}$ à La Rochelle jusqu'à $17 \mathrm{~cm}$ à la pointe de Gatseau. Les écarts plus forts en ce point sont vraisemblablement dus à une mauvaise représentation de la bathymétrie qui y est très changeante.

Les écarts entre le modèle et les observations réalisées au point S4, pour la période du 2 au 27 novembre 2002 (Tableau 1) montrent une représentation des hauteurs d'eau et des vitesses, comparable aux écarts trouvés dans la littérature 
(STANISIERE et al., 2006). Le modèle représente bien les oscillations semidiurnes et les variations d'amplitudes des effets de la marée aussi bien en hauteur d'eau qu'en courant, les erreurs proviennent principalement des écarts de phase (Figure 5a,b et c).

En termes de reproduction de circulations induites par le vent, les surcotes et les courants (induits par le vent) calculés par le modèle sont comparés aux observations du courantomètre S4 en novembre 2002, en extrayant les informations de surcote et de courant résiduel (signal brut moins signal de marée) grâce au logiciel T-Tide. Bien que les erreurs calculées soient fortes par rapport au phénomène représenté (de l'ordre de 20\%, voir Tableau 1), les tendances des surcotes sont bien respectées, ainsi que les effets du vent sur les courants (Figure $5 \mathrm{~d}$ et e).

\begin{tabular}{|l|c|c|}
\hline & Erreur quadratique moyenne & Max. mesuré \\
\hline Cote de surface libre de marée $(\mathrm{m})$ & 0.15 & 3 \\
\hline Surcote $(\mathrm{m})$ & 0.10 & 0.42 \\
\hline Courant de marée $(\mathrm{m} . / \mathrm{s})$ & 0.09 & 0.62 \\
\hline Courant résiduel $(\mathrm{m} / \mathrm{s})$ & 0.06 & 0.35 \\
\hline
\end{tabular}

Tableau 1 - Ecarts entre mesures $S 4$ et modèle.
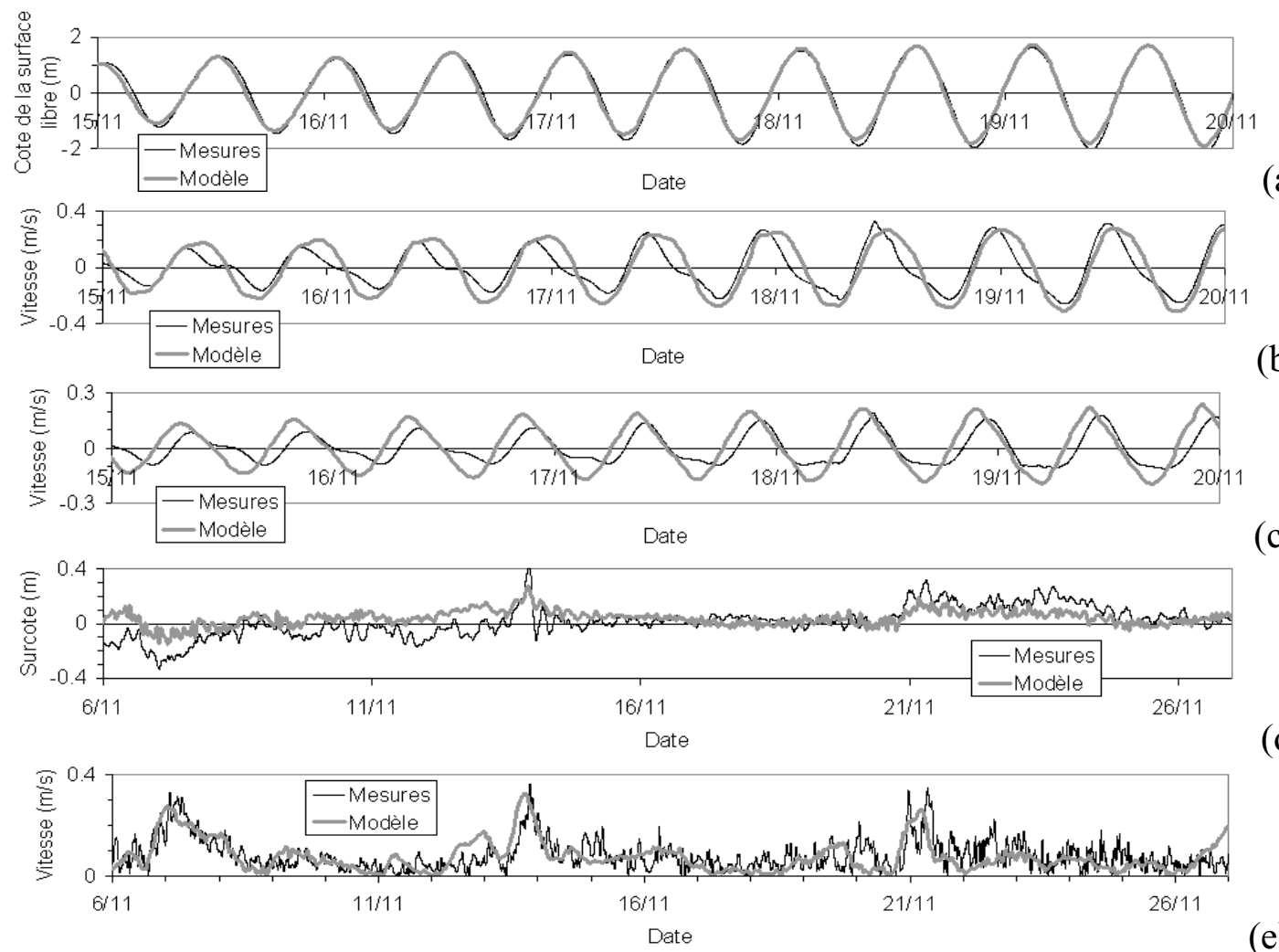

Figure 5 - Comparaison modèle-mesure S4 (a) niveaux d'eau de marée, (b) courant de marée zonal, (c) courant de marée méridien, (d) surcotes, (e) courants résiduels. 


\section{$5 \quad$ Formulation théorique de la mobilité sédimentaire}

\subsection{Mise en mouvement}

Dans cette étude, la méthodologie proposée par IDIER et al., (2006) est utilisée. Pour qu'un sédiment soit mis en mouvement, la contrainte au fond doit être supérieure à une contrainte critique. Afin d'estimer les tailles maximales de grains mis en mouvement par l'action des courants et des vagues, nous utilisons la valeur du paramètre de Shields pour la mise en mouvement de sédiments de tailles $\mathrm{d}_{50}$ :

$$
\theta=\frac{\tau_{b}}{g\left(\rho_{s}-\rho\right) d_{50}}
$$

$\tau_{b}\left(\mathrm{~N} / \mathrm{m}^{2}\right)$ est la contrainte hydrodynamique appliquée sur le fond. Cette contrainte peut être exercée par le courant (cisaillement au fond dû aux courants, $\tau_{b, c}$ ), par la houle (contrainte au fond due aux vagues, moyennée sur un demicycle de vagues, $\left.\tau_{b, w}\right)$. D'après VAN RIJN (1989), la valeur absolue de la contrainte $\tau_{b, c w}$ exercée au fond sous l'action conjointe des courants et des vagues est de la forme $\left|\tau_{b, c w}\right|=\alpha_{r} \tau_{b, c}+\left|\tau_{b, w}\right|$ avec $\alpha_{r}$ le coefficient de réduction de contrainte au fond $(\leq 1)$, qui est largement inférieur à 1 en présence de vagues relativement fortes conjuguées avec de faibles courants. L'estimation de ce coefficient nécessite la connaissance de la rugosité apparente et de l'épaisseur des couches limites. En première approche nous prenons $\alpha_{r}=1$, ce qui ne semble pas aberrant étant donné que les courants de marée sont relativement importants dans la zone. Ceci implique que la contrainte totale est potentiellement légèrement surestimée dans les résultats présentés dans cet article.

Après avoir estimé ainsi la contrainte totale, à partir des paramètres de Shields critiques, nous déduisons la taille maximale des grains mis en mouvement sous cette contrainte. Pour la mise en mouvement, seules les contraintes liées à la rugosité de peau sont considérées. En outre, le domaine d'étude est ici restreint aux sédiments non-cohésifs $\left(d_{50}>0,063 \mathrm{~mm}\right)$. Ainsi, sur les figures, les zones où la couverture sédimentaire est de nature cohésive sont hachurées.

\subsection{Contraintes hydrodynamiques}

La contrainte au fond due aux vagues $\left(\tau_{b, w}\right)$ et moyennée sur un demi-cycle de vagues, s'exprime sous la forme (VAN RIJN, 1989): $\tau_{b, w}=0.25 \rho f_{w}\left(\hat{u}_{b}\right)^{2}$ où $\hat{u}_{b}$ est la valeur pic de la vitesse orbitale près du fond, suivant la théorie linéaire. $f_{w}$ est le coefficient de frottement des vagues qui est fonction de la longueur d'excursion des vagues, elle-même fonction de la valeur pic de la vitesse orbitale et de la période pic des vagues. Les paramètres issus du modèle de vague utilisés pour calculer la mobilité sont donc : la période pic et la vitesse orbitale maximale en chaque nœud du maillage, à chaque instant. La contrainte de cisaillement au fond due aux courants est donnée par l'expression $\tau_{b}=\rho g U^{2} /\left(K . h^{1 / 6}\right)^{2} . U(\mathrm{~m} / \mathrm{s})$ 
est la norme de la vitesse horizontale moyennée sur la verticale, $K$ le coefficient de frottement de Strickler, et $h(\mathrm{~m})$ la hauteur d'eau. Les paramètres issus du modèle de courant utilisés pour calculer la mobilité sont donc : la hauteur d'eau et l'intensité des courants en chaque nœud du maillage, à chaque instant.

\section{$6 \quad$ Mobilité des sédiments non-cohésifs}

Les tailles de grain mises en mouvement obtenues par modélisation au point de mesure S4 situé sur le seuil interinsulaire montrent un accord acceptable (écart de $20 \%$ ) avec les tailles estimées à partir des mesures hydrodynamiques (IDIER et al., 2006) . Ceci justifie l'utilisation du modèle pour étendre spatialement les résultats obtenus à partir de mesures hydrodynamiques dans (IDIER et al., 2006).

La Figure 6a montre la taille maximale des grains potentiellement mis en mouvement en chaque nœud des modèles, indépendamment de la nature de la couverture sédimentaire, en supposant une rugosité de fond due à une taille de grain de $0,2 \mathrm{~mm}$. Les résultats montrent que les grains sont préférentiellement mis en mouvement sur la partie externe des Pertuis, avec des tailles atteignant $2 \mathrm{~mm}$ par $30 \mathrm{~m}$ de fond et $4 \mathrm{~mm}$ le long des façades Atlantique des deux îles. Entre les îles et le continent, les tailles mobilisées sont nettement plus faibles, de l'ordre de 0,5 à $1 \mathrm{~mm}$. La Figure $6 \mathrm{~b}$ montre que la mise en mouvement, au large, et le long des façades Atlantique des îles est principalement due aux vagues, tandis que, d'après le modèle, seuls les endroits de fort courant (pointes Nord des iles, Pertuis Breton, Pertuis de Maumusson) permettent une mise en mouvement significative du sédiment sous l'action du courant. La plupart de ces zones sont peu soumises à la houle et donc seul le courant y aurait un effet sur la mise en mouvement. Notons toutefois que, dans certaines zones à proximité du Pertuis de Maumusson, les courants induits par les vagues sont non négligeables (BERTIN et al., 2008). Dans une étape ultérieure il faudrait donc prendre en compte ce type de courants.

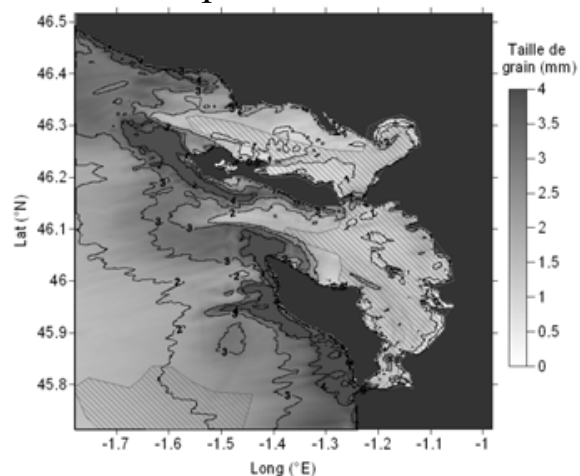

\section{Figure 6 - (a) Taille de grain maxima}

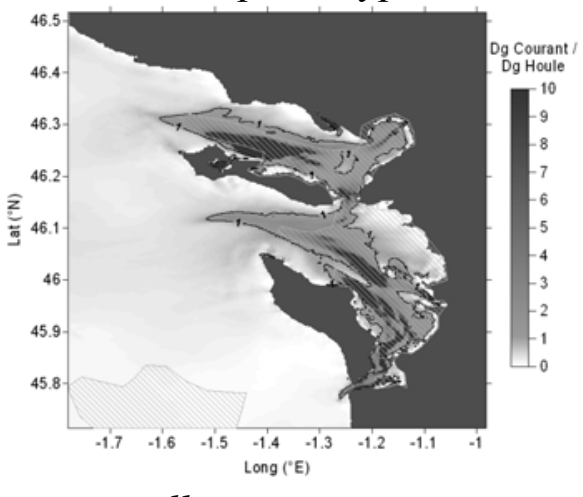

(b)

Figure 6 - (a) Taille de grain maximale potentiellement mise en mouvement (période 5-27 nov. 2002) par les courants et les vagues. (b) Rapport entre tailles de grain mises en mouvement par les courants et par les vagues. Les zones hachurées représentent de manière schématique les zones de vase. 
Afin d'estimer une mobilité représentative de toute la période, les temps relatifs de mise en mouvements de grains de $0,2 \mathrm{~mm}$ sont calculés. D'après la Figure 7a, ces grains seraient mis en mouvement pendant $80 \%$ de la période étudiée dans une majeure partie de la zone d'étude (façades Atlantique des îles). Ils seraient peu mis en mouvement $(\sim 50 \%)$ au Sud-Ouest (prof. de 40-50 m), et encore moins dans la zone interne des Pertuis $(<50 \%)$. Ceci est cohérent avec les résultats sur les tailles de grains maximales (Figure 6a). La figure $8 \mathrm{~b}$ montre que les vagues, dont l'action est dominante sur la partie externe des Pertuis, y engendreraient la mise en mouvement des grains pendant la majeure partie de la période étudiée. En revanche (Figure 7c), le courant, dont l'action est dominante dans la zone interne, y engendrerait la mobilité du sédiment pendant deux fois moins de temps.
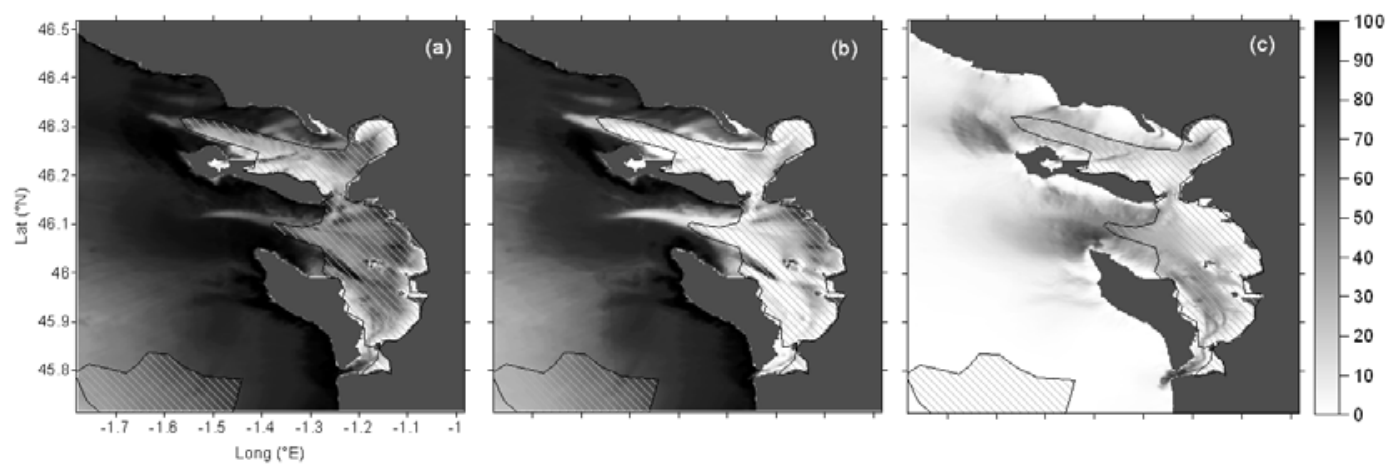

Figure 7 - Temps relatifs de mise en mouvement de grain de 0,2 $\mathrm{mm}$ sur la durée de la campagne de mesure (5-27 nov. 2002) sous l'action: (a) des courants et des vagues, (b) des vagues, (c) des courants. Les zones hachurées représentent de manière schématique les zones de vase.

\section{$7 \quad$ Conclusions et perspectives}

La présente étude s'appuie sur des travaux de modélisation basés sur un certain nombre d'hypothèses (nature du fond supposée uniforme, interactions vaguescourants négligées). Cependant, elle met en évidence l'importance des houles dans la dynamique d'une plateforme continentale interne comme le site des Pertuis Charentais, en dehors des problématiques de courants de houle. A partir de modèles de vagues et courants validés, et appliqués pour la période du mois de novembre 2002, il est mis en évidence que, pendant cette période, des tailles de grains allant jusqu'à $4 \mathrm{~mm}$ pourraient être mises en mouvement et que des grains de $0,2 \mathrm{~mm}$, sur les façades Atlantique des îles seraient transportés pendant plus de $80 \%$ du temps. A l'Ouest des îles, l'action des vagues apparait dominante pour la mise en mouvement, tandis qu'à l'Est et dans les Pertuis, l'action des courants apparait dominante.

Dans cette étude, les vagues et les courants (marée, tempête) sont pris en compte séparément et les courants de houles sont négligés. Or, dans des zones comme le 
Pertuis de Maumusson, les courants de houle ne sont pas négligeables devant les courants de marée ou d'onde de tempête. Il conviendrait donc de les prendre en compte et d'étudier leur influence sur la mobilité des sédiments.

\section{Remerciements}

Les auteurs remercient l'IFREMER (en particulier Franck Dumas) de les avoir autorisés d'utiliser le code MARS pour ce projet, ainsi que Sophie Lecacheux (BRGM) pour sa contribution.

\section{Références bibliographiques}

1 BARTHE X., CASTAING P, (1989) Étude théorique de l'action des courants de marée et des houles sur les sédiments du plateau continental du golfe de Gascogne, Oceanol. Acta 12 (4) 325-334.

2 BERTIN X., CHAUMILLON E., SOTTOLICHIO A. and PEDREROS R., (2005), Tidal inlet response to sediment infilling of the associated bay and possible implications of human activities: the Marennes-Oléron Bay and the Maumusson Inlet, France, Continental Shelf Research, 25, 1115-1131.

3 BERTIN X., CASTELLE B., CHAUMILLON E., BUTEL R., QUIDUE R. (2008), Longshore transport and inter-annual variability at a high energy dissipative beach: St Trojan, SW Oléron Island, France, Continental Shelf Research, accepted.

4 BOOIJ N., HAAGSMA IJ.G., HOLTHUIJSEN L.H., KIEFTENBURG A.T.M.M., RIS R.C., van der WESTHUYSEN A.J. and ZIJLEMA M., (2004). Swan Cycle III version 40.41. User's Manual, 115p.

5 IDIER D., PEDREROS R., OLIVEROS C., SOTTOLICHIO A., CHOPPIN L. et BERTIN X., (2006), Contributions respectives des courants et de la houle dans la mobilité sédimentaire d'une plateforme interne estuarienne. Exemple: le seuil interinsulaire, au large $d u$ Pertuis d'Antioche, France. C.R. Geoscience, Vol. 338, 718-726.

6 LYARD F., F. LEFEVRE, T. LETELLIER and O. FRANCIS, (2006), Modelling the global ocean tides: modern insights from FES2004, Ocean Dynamics, DOI 10.1007/s10236-006-0086-x.

7 PEDREROS R., BRUNEAU N., BONNETON P., DUMAS F. and IDIER D. (2006). Hydrodynamics and morphodynamic evolution of the nearshore zone using the coupling of SWAN, MARS and a sedimentary transport module. Proc. Operational Coastal Oceanography Conference, Brest 16-17 October.

8 SHOM, (2000), Carte spéciale $\mathrm{G} \mathrm{n}{ }^{\circ} 6333 \mathrm{G}$.

9 STANISIERE J.Y., DUMAS F., PLUS M., MAURER D., ROBERT S., (2006) Caractérisation des composantes hydrodynamiques d'un système côtier semifermé : Le Bassin de Marennes-Oléron, Rapport Ifremer Ref DOP/LER.LER/PC/06.1059.

10 Van RIJN L.C., (1989), Handbook of sediment transport by currents and waves. Report H461, WL|Delft Hydraulics, The Netherlands. 